\title{
Chromosome Centromeres: Structural and Analytical Investigations with High Resolution Scanning Electron Microscopy in Combination with Focused Ion Beam Milling
}

\author{
E. Schroeder-Reiter G. Wanner
}

Department Biology I, Ultrastructural Research, Biozentrum der Ludwig-Maximilians-Universität München, Planegg-Martinsried, Germany

\section{Key Words \\ CENH3 - Centromere ultrastructure - Chromosomes • \\ FESEM/FIB • Field emission scanning electron microscopy · \\ Focused ion beam milling $\cdot \mathrm{H} 3 \mathrm{~S} 10 \mathrm{ph}$}

\begin{abstract}
Whole mount mitotic metaphase chromosomes of different plants and animals were investigated with high resolution field emission scanning electron microscopy (FESEM) to study the ultrastructural organization of centromeres, including metacentric, acrocentric, telocentric, and holocentric chromosome variants. It could be shown that, in general, primary constrictions have distinctive ultrastructural features characterized by parallel matrix fibrils and fewer smaller chromomeres. Exposure of these structures depends on cell cycle synchronization prior to chromosome isolation, chromosome size, and chromosome isolation technique. Chromosomes without primary constrictions, small chromosomes, and holocentric chromosomes do not exhibit distinct ultrastructural elements that could be directly correlated to centromere function. Putative spindle structures, although rarely observed, spread over the primary constriction to the bordering pericentric regions. Analytical FESEM techniques, including specific DNA staining with Pt blue,
\end{abstract}

staining of protein as a substance class with silver-colloid, and artificial loosening of fixed chromosomes with proteinase $\mathrm{K}$, were applied, showing that centromere variants and ultrastructural elements in the centromere differ in DNA and protein distribution. Immunogold localization allowed highresolution comparison between chromosomes with different centromere orientations of the distribution of centromere-related histone variants, phosphorylated histone $\mathrm{H} 3$ (ser10), and CENH3. A novel application of FESEM combined with focused ion beam milling (FIB) provided new insights into the spatial distribution of these histone variants in barley chromosomes.

Copyright $\odot 2009$ S. Karger AG, Basel

For investigation of the higher order structure of chromatin, field emission scanning electron microscopy (FESEM) has proven to be a valuable tool since it allows high resolution imaging of chromosome structures down to the dimension of the $10 \mathrm{~nm}$ elementary fibril. Techniques for isolation of chromosomes under conditions allowing greatest possible structural preservation have been developed and applied in the past decade [Schubert et al., 1993; Martin et al., 1994]. This has made the visualization of basic structural elements of chromosomes,

\section{KARGER}

Fax +4161306 1234

E-Mail karger@karger.ch

www.karger.com
C) 2009 S. Karger AG, Basel

$1424-8581 / 09 / 1244-0239 \$ 26.00 / 0$

Accessible online at:

www.karger.com/cgr
Gerhard Wanner

Department Biology I, Ultrastructural Research

Biozentrum der Ludwig-Maximilians-Universität München

Großhadernerstrasse 2-4, DE-82152 Planegg-Martinsried (Germany)

Tel. +49 892180 74400, Fax +4989 2180 74415, E-Mail wanner@lrz.uni-muenchen.de 
matrix fibrils, and chromomeres possible [Martin et al., 1996; Wanner and Formanek, 2000; Zoller et al., 2004], which so far have proven to be universal for all chromosomes investigated. To determine the composition of structural elements, staining and labeling techniques have been applied: platinum $(\mathrm{Pt})$ blue for specific labeling of DNA, silver-compounds for labeling of protein as a substance class, and immunogold labeling of specific DNA sequences and proteins as well as their epigenetic modifications [Wanner and Formanek, 1995, 2000; Schroeder-Reiter et al., 2003, 2006; Houben et al., 2007; Metcalfe et al., 2007]. Determining basic structural elements of centromeres as essential structures of eukaryotic chromosomes is of high priority, but despite significant advances in the understanding of chromosome architecture, the ultrastructural characterization of the centromere in a functional context has remained elusive.

Scanning electron microscopy (SEM) investigations on human, Chinese hamster, rye, and barley chromosomes have shown that centromeres in mitotic metaphase have a predominance of parallel fibers as common structural characteristics [Harrison et al., 1982; Wanner et al., 1991; Sumner, 1998; Zoller et al., 2004]. Studies have unveiled centromere-specific histone variants and/or posttranslational histone modifications, including phosphorylated histone $\mathrm{H} 3$ serine 10 (H3S10ph), which seems to be involved in sister chromatid cohesion in plants [Kaszas and Cande, 2000; Manzanero et al., 2000], and CENH3, a centromere-specific histone $\mathrm{H} 3$ variant which is fundamental in kinetochore and spindle apparatus assembly [Talbert et al., 2002, 2004; Black and Bassett, 2008]. Recently, distributions of 3 histone $\mathrm{H} 3$ variants have been compared with FESEM in barley chromosomes, revealing centromere-specific patterns for unmodified histone H3, H3S10ph, and CENH3 located to chromosome substructures [Schroeder-Reiter et al., 2003; Houben et al., 2007]. FESEM in combination with focused ion beam (FIB) applications are relatively new for biological studies [Drobne et al., 2007; Knott et al., 2008] but present a promising possibility for getting insight into biological substructures. The present study aims to structurally characterize centromere variants to determine whether there are conserved centromere structures (i.e., parallel fibrils) for a variety of organisms. Using high resolution FESEM labeling techniques, including differential substance class detection, immunogold labeling, and FIB milling, the substructural composition and spatial distribution of functional elements in centromeres were investigated on a nanometer scale.

\section{Materials and Methods}

Plant and Animal Material

Chromosomes studied were isolated from the following organisms: Hordeum vulgare cv. Steffi (barley; BayWa AG, Germany); Secale cereale (rye; BayWa AG, Germany); Silene latifolia (root tips were provided by A. Houben, IPK, Gatersleben, Germany); Homo sapiens (chromosome suspensions were provided by J. Murken); Vicia faba (chromosomes were provided by A. Houben, IPK, Gatersleben, Germany); Oziroë biflora (root tips were provided by J. Grau, Geo-Bio Center, Munich, Germany) [see Guaglianone and Arroyo-Leuenberger, 2002]; wheat-barley (Triticum aestivum cv. Chinese Spring and Hordeum vulgare cv. Betzes) addition line (seeds provided by T. Endo, Kyoto, Japan) [see Nasuda et al., 2005]; Luzula nivea (Ernst Benary Seeds, Hann.-Muenden, Germany); Luzula elegans (seedlings were provided by A. Houben, IPK, Gatersleben); Macropus rufogriseus $\times$ Macropus agilis (wallaby hybrid chromosomes provided by $\mathrm{R}$. O’Neill, UConn, CT, USA) [see Metcalfe et al., 2007].

\section{Chromosome Fixation and Isolation}

Divisions of root-tip cells of sprouting seeds were fixed with 3:1 (v/v) ethanol:acetic acid. If cell cycle was synchronized, cells were arrested at metaphase before fixation. Chromosomes were isolated by maceration with cellulase/pectolyase from root-tip cells and were dropped onto glass slides according to the drop/ cryo technique [Martin et al., 1994]. Alternatively, root tips were fixed (2\% formaldehyde in $10 \mathrm{mM}$ Tris, $10 \mathrm{mM} \mathrm{Na}_{2}$ EDTA, $100 \mathrm{mM}$ $\mathrm{NaCl}, \mathrm{pH}$ 7.5) and mechanically dispersed (Polytron ${ }^{\circledR} 5 \mathrm{~mm}$ mixer, Kinematica, Switzerland) in isolation buffer ( $15 \mathrm{~mm}$ Tris, $2 \mathrm{mM}$ $\mathrm{Na}_{2}$ EDTA, $0.5 \mathrm{~mm}$ spermin, $80 \mathrm{mM} \mathrm{KCl}, 20 \mathrm{~mm} \mathrm{NaCl}, 15 \mathrm{~mm}$ mercaptoethanol, 1\% Triton-X-100). The resulting suspension was centrifuged with a swing-out rotor onto laser marked slides [for details see Schubert et al., 1993].

\section{DNA Staining with Pt Blue}

For DNA staining, chromosomes were stained for $30 \mathrm{~min}$ at room temperature with platinum blue $\left(\left[\mathrm{CH}_{3} \mathrm{CN}\right]_{2} \mathrm{Pt}\right.$ oligomer, 10 $\mathrm{mM}$ in aqua bidest., $\mathrm{pH}$ 7.2) and subsequently washed with distilled water [for details see Wanner and Formanek, 1995].

\section{Artificial Decondensation}

Glutardialdehyde-fixed chromosomes were washed in buffer, blocked with $1 \%$ glycine solution, and incubated with proteinase $\mathrm{K}(1 \mathrm{mg} / \mathrm{ml})$ for $2 \mathrm{~h}$ at $37^{\circ} \mathrm{C}$ [for details see Wanner and Formanek, 2000].

\section{Protein Staining with Ag-Colloid}

For silver staining, chromosomes were stained with an aqueous solution of colloidal silver containing $0.1 \mathrm{M}$ elementary silver at pH 8 [for details see Wanner and Formanek, 2000].

\section{Immunogold Labeling}

Chromosome preparations were washed in PBS buffer, blocked in $1 \%$ bovine serum albumin and $0.1 \%$ Tween 20 in PBS for 30 $\mathrm{min}$, and incubated with the respective primary antibody (diluted in blocking solution) for $1 \mathrm{~h}$ (1:250 rabbit anti-serine 10 phosphorylated histone $\mathrm{H} 3$, Upstate Biotechnologies; 1:300 rabbit anti-OsCENH3 courtesy of P. Talbert) [see Nagaki et al., 2004]. After washing, specimens were incubated with anti-rabbit Nano- 
gold $^{\circledR}$ Fab'-fragments (Nanoprobes) for $1 \mathrm{~h}$. Specimens were washed and post-fixed with $2.5 \%$ glutardialdehyde in PBS and subsequently silver enhanced (HQ Silver, Nanoprobes) according to the manufacturer's instructions.

\section{Field Emission Scanning Electron Microscopy (FESEM)}

Prior to SEM, specimens were washed in distilled water, dehydrated in acetone, critical point dried from $\mathrm{CO}_{2}$, cut to size, and mounted onto aluminum stubs. Specimens were sputter-coated with platinum or carbon-coated by evaporation (Balzers high vacuum evaporator BAE 121, Liechtenstein) to a layer of 3-5 nm, and examined - dependent on chromosome size and analytical conditions - between $3 \mathrm{kV}$ and $30 \mathrm{kV}$ with a Hitachi S-4100 field emission scanning electron microscope equipped with a YAGtype backscattered electron (BSE) detector (Autrata). Secondary electron (SE) and BSE images were recorded simultaneously with DigiScan $^{\mathrm{TM}}$ hardware and processed with Digital Micrograph 3.4.4 software (Gatan, Inc. Pleasanton).

\section{FESEM Combined with Focused Ion Beam Milling}

(FESEM/FIB)

The FIB work was performed by means of 2 FESEM/FIB systems, a Zeiss Neon 1540 EsB and a Zeiss NVision 40. For both systems the focused ion beam consists of $\mathrm{Ga}^{+}$ions accelerated by a voltage of $30 \mathrm{kV}$. Images were recorded using in-lens SE, in-lens energy selective backscattered (EsB), and chamber EverhardThornley SE detectors. The SEM column is mounted on top of the chamber and the FIB column at an angle of $54^{\circ}$.

\section{Results}

\section{Centromere Structure with FESEM}

Mitotic metaphase chromosomes with various centromere positions were investigated with FESEM. Chromosomes were considered to be in metaphase when a primary constriction was recognizable [Martin et al., 1996; Zoller et al., 2004]. At lower magnifications, centromere positions ranging from metacentric (barley, rye, Silene latifolia, human, Ozirö̈ biflora) to acrocentric (Vicia faba, Oziroë biflora, human) could be recognized as primary constrictions varying in length (fig. 1A-E). In metaphase spreads from O. biflora 32 chromosomes (2n) range in size from $1.8 \mu \mathrm{m}$ to $10 \mu \mathrm{m}$, allowing direct comparison of different sized chromosomes in identical stages of condensation. The centromere was obvious in large and mid-sized chromosomes; chromosomes less than $2 \mu \mathrm{m}$ in length appeared approximately spherical at all magnifications, without an obvious primary constriction (fig. 1F). No constriction was visible in telocentric chromosomes from a wheat-barley addition line; the centromere was only structurally recognizable in late metaphase as a slightly tapered end terminal to separated sister chromatids (fig. 1G). Holocentric chromosomes of the genus Luzula exhibited, as expected per definition, no primary constriction, even at moderate magnifications (fig. $1 \mathrm{H}$ ). In contrast to other chromosomes studied, metaphase holocentric chromosomes of Luzula nivea and Luzula sylvatica in particular were characteristically interconnected by a fibrillar network, presumably of nucleoplasmic origin. Chromosomes used for routine investigations are usually synchronized and arrested, which precludes formation of the spindle apparatus. However, even under conditions conducive to maintenance of spindle elements, by which chromosomes are isolated from formaldehyde-fixed (non-arrested) root tip cells, fibrillar structures attached to the primary constriction, and immediately bordering pericentric regions were only rarely observed (fig. 1J).

Regardless of the centromeric position, all chromosomes studied exhibited rather compact chromomeres on the chromosome arms. The chromatin in the centromere, however, differs structurally from that of chromosome arms and is characterized by parallel matrix fibrils, with either few small chromomeres or without chromomeres (fig. 2A). In small chromosomes without primary constrictions parallel fibers were not visible (fig. 1F). Telocentric centromeres, although tapered, exhibited no parallel fibrils (fig. 1G). In the case of holocentric chromosomes, chromomeres are interspersed with parallel fibrils along the entire chromosome (fig. 2B). The length and width of the primary constriction is influenced by the treatment prior to and during chromosome isolation; synchronized chromosomes tend to exhibit a more pronounced constriction with parallel fibrils than non-synchronized chromosomes, and chromosomes isolated by dispersion and centrifugation exhibit significantly longer 'stretched' centromeric regions, often with small chromomeres (fig. 2C, D).

Metaphase chromosomes stained for DNA with $\mathrm{Pt}$ blue typically revealed weaker signals at the primary (and secondary) constrictions and along the longitudinal axis defining sister chromatids (fig. 3A). Holocentric chromosomes in early metaphase occasionally exhibited a weak $\mathrm{Pt}$ blue signal along the longitudinal chromosome axis (fig. 3B) which disappears in late metaphase when chromosomes become extremely compact. Some wallaby (marsupial) hybrid chromosomes exhibit strikingly long centromeres and show particularly well the dominant presence of parallel fibrils interspersed with more compact chromatin with a strong $\mathrm{Pt}$ blue signal, indicating a high DNA content (fig. 3C).

For further insight into DNA distribution in compact regions, Pt blue staining and artificial loosening of (glu- 

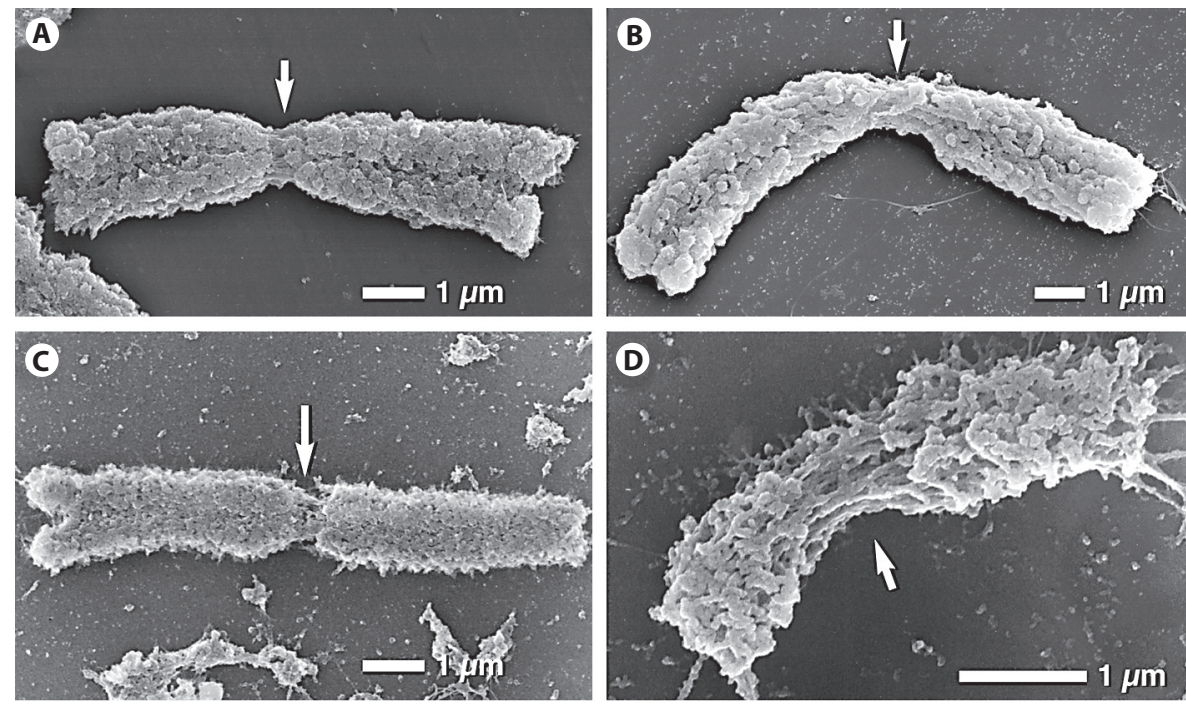

Fig. 1. Scanning electron micrographs of mitotic metaphase chromosomes from different organisms with centromere variants, including metacentric, acrocentric, telocentric, and holocentric orientations. Secondary electron images of chromosomes with interstitial centromeres show that topography of primary constriction (arrows) is different from that of the chromosome arms. A Barley (Hordeum vulgare) metacentric chromosome. B Rye (Secale cereale) metacentric chromosome. C Silene latifolia metacentric chromosome. D Human submetacentric chromosome. E Vicia faba acrocentric chromosome. F Small chromosome from Oziroë biflora without a recognizable primary constriction. G Telocentric chromosome from a wheat-barley addition line with a small terminal tapering (asterisk) opposite the telomeric end. $\mathbf{H}$ Holocentric chromosome from Luzula nivea without primary constriction. J Fibrillar structures are occasionally observed on non-synchronized barley chromosomes isolated by dispersion and centrifugation and are attached to both the primary constriction and the bordering pericentric regions.
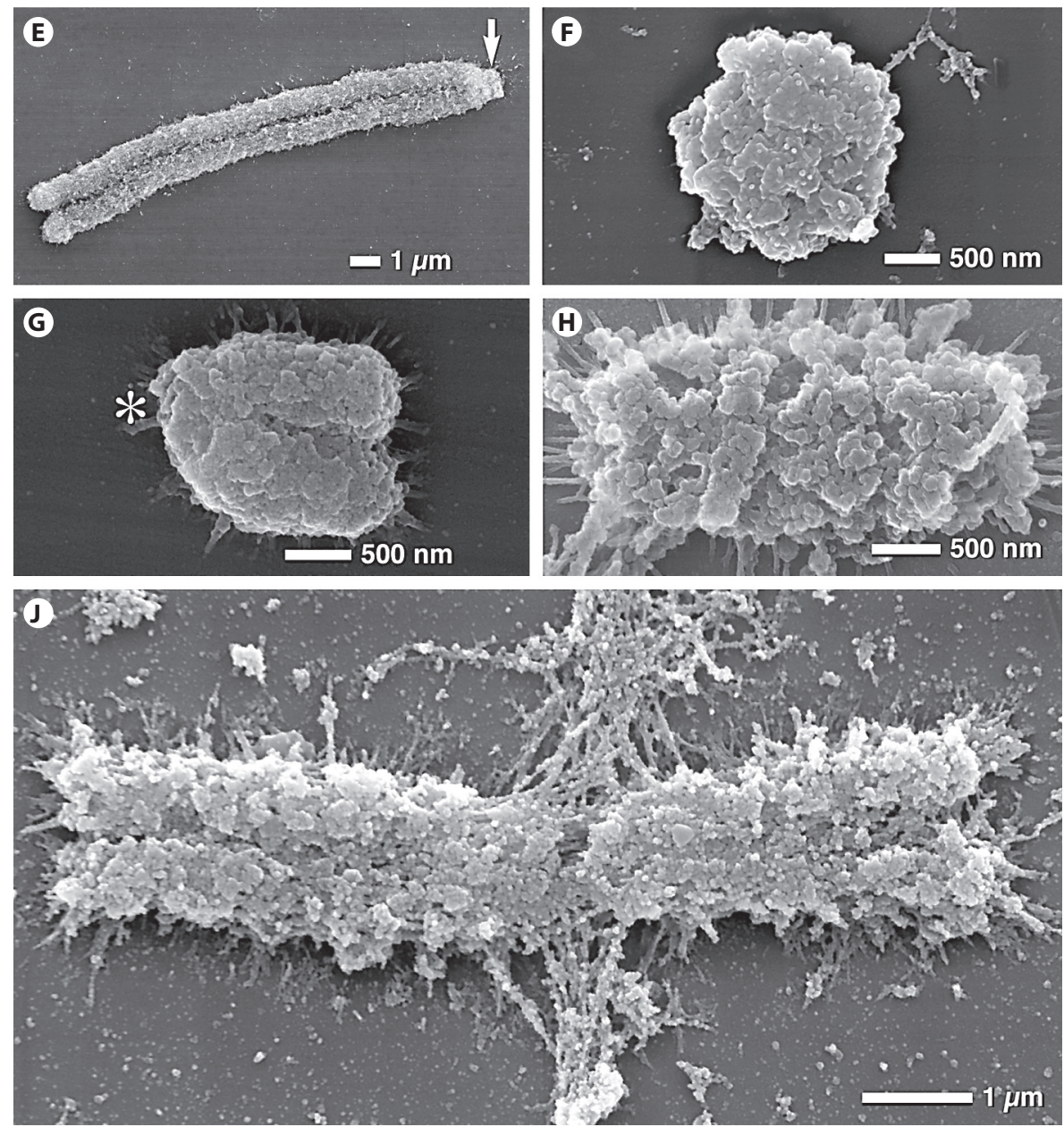

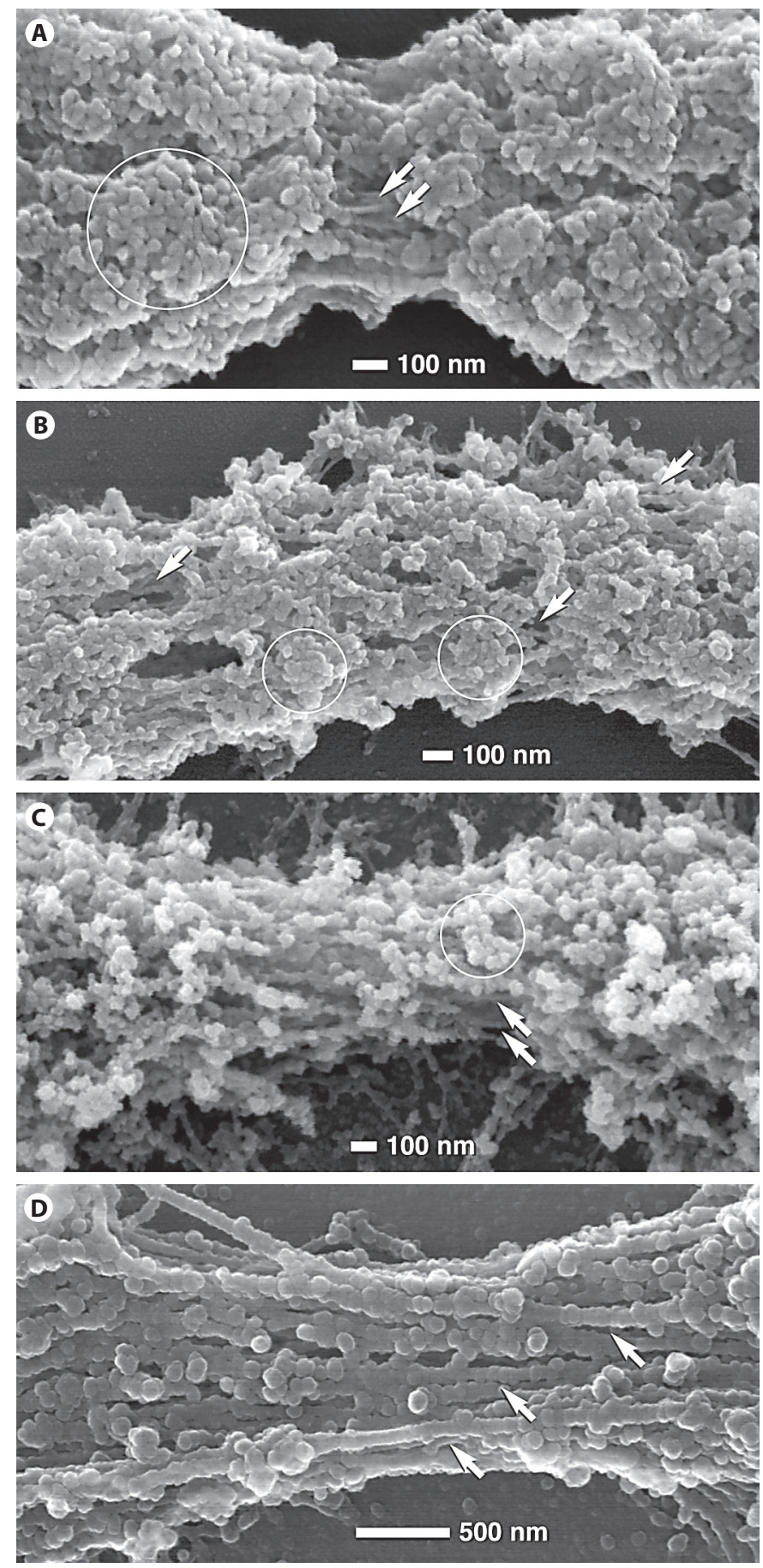

Fig. 2. Scanning electron micrographs showing characteristic structural elements of interstitial centromeres and holocentric chromosomes. A Centromere of a barley chromosome isolated by the drop/cryo method exhibits parallel fibrils (arrows), with few chromomeres at the primary constriction, which is characteristic for interstitial centromeres, bordered by compact chromomeres (circle) on the chromosome arms. B Holocentric Luzula nivea chromosomes exhibit chromomeres (circles) interspersed with tardialdehyde-fixed) chromosomes with proteinase K revealed various higher order structures of chromatin fibrils ranging from loosened chromomeres down to 30 $\mathrm{nm}$ fibrils distributed throughout the chromosome arms and centromere (fig. 3D). The parallel character of chromatin in centromere regions becomes more pronounced, and a continuity between parallel elements in the centromere and those in the chromosome arms is obvious (fig. 3D). On non-arrested chromosomes isolated from formaldehyde-fixed root tips, fibrillar structures radiating from the centromeric region show strong topographic contrast and stain in part with Pt blue (fig. 3E). Despite the high proportion of protein in chromosome composition (approx. two-thirds of total mass) [Earnshaw, 1988], Ag-colloid staining for protein as a substance class resulted in only a moderate signal along the chromosome arms and more intense staining of the centromere compared to Pt blue (fig. 3F).

\section{Immunolabeling for Localization of Histone Variants}

Two histone variants, phosphorylated histone $\mathrm{H} 3$ serine $10(\mathrm{H} 3 \mathrm{~S} 10 \mathrm{ph})$ and $\mathrm{CENH} 3$, a centromere-localized histone $\mathrm{H} 3$ variant, were immunolocalized to metaphase chromosomes from different organisms to facilitate identification of their respective functional centromere regions and to determine their centromere-specific signal distribution with high resolution. The pericentric distribution of H3S10ph in plants was confirmed for metacentric and acrocentric chromosomes showing symmetric labeling on either side of the centromere (fig. 4A, B). In small acrocentric chromosomes of Oziroë, the entire short arm was labeled (fig. 4C). In addition, in Oziroë chromosome complements containing different sized chromosomes, labeled regions were equal-sized, regardless of the relative length of the chromosome arms (fig. 4D). Although small chromosomes were labeled nearly entirely (fig. 4D), labeling with H3S10ph exhibited an axial symmetry in its labeling intensity (fig. 4D). Telocentric chromosomes of the wheat-barley addition line

parallel fibrils (arrows) along the entire chromosome arms. C, D Chromosomes isolated by dispersion and centrifugation often exhibit elongated centromeres, resulting either in the presence of small chromomeres (circle) over the primary constriction with exposed parallel fibers (arrows), as shown for metacentric barley centromeres (C) or highly exposed 'stretched' parallel fibrils (arrows), as is shown for an acrocentric centromere from Vicia faba (D). 

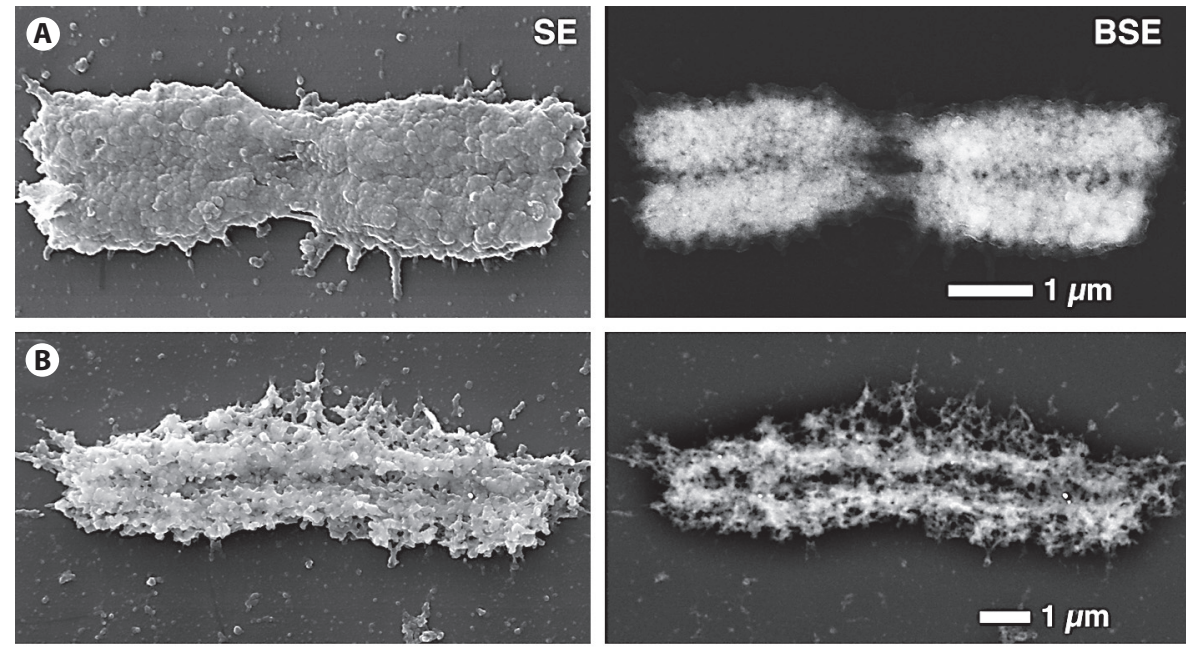

Fig. 3. Scanning electron micrographs of chromosomes from various organisms after staining for DNA or protein as substance classes showing chromosome topography (secondary electron images = SE, left column) and signal distribution (backscattered electron images = BSE, right column). A Specific DNA staining with Pt blue in barley chromosomes shows a weak signal in the centromere typical for interstitial centromeres. Sister chromatids are clearly distinguishable in the BSE image (right). B Luzula elegans holocentric chromosomes stained with Pt blue exhibit a clear axial separation of chromatin, presumably of sister chromatids, only in early metaphase. C Hybrid wallaby (marsupial) chromosomes have elongated centromeres which exhibit both parallel fibrils and loosened chromomeres (left) and a strong Pt blue signal (right) indicating a high overall DNA content, with some fibrils containing little DNA (arrows). D Centromeric region of a barley chromosome artificially loosened with proteinase $\mathrm{K}$ and stained with Pt blue shows a continuity of parallel elements extending over the centromere into the chromosome arms. Some fibrils do not stain with Pt blue, indicating that they contain little or no DNA (arrows). E A low proportion of fibrillar structures attached on centromeres of non-synchronized barley chromosomes isolated by dispersion and centrifugation exhibits signal traces from Pt blue (arrows). F Under stringent conditions, staining with colloidal silver results in a stronger signal in the centromere, here barley, than in the chromosome arms, indicating that the centromere is protein-enriched.
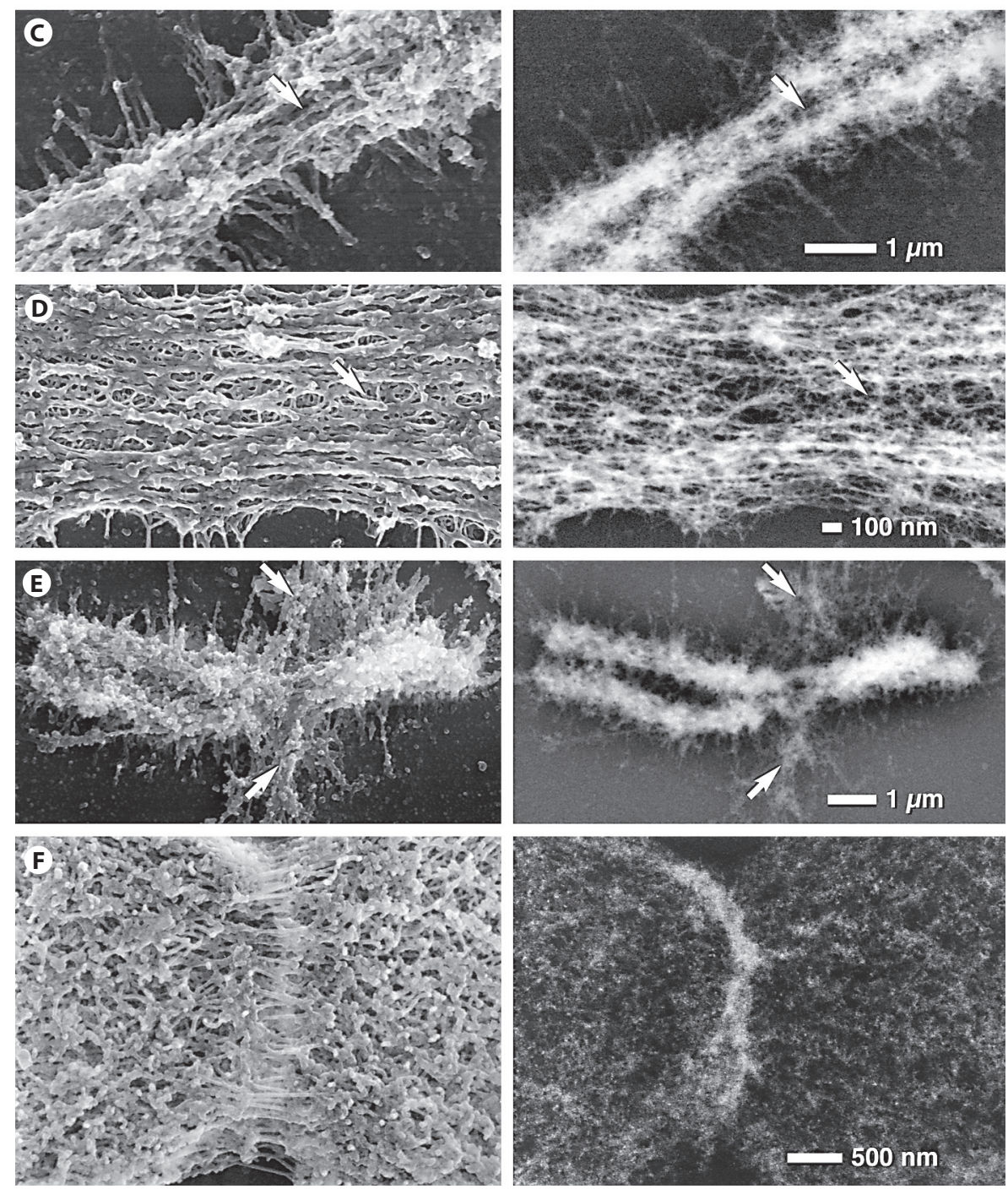
Fig. 4. Scanning electron micrographs of chromosomes from various organisms after immunogold labeling for phosphorylated histone $\mathrm{H} 3$ at serine $10(\mathrm{H} 3 \mathrm{~S} 10 \mathrm{ph})$ showing chromosome topography (secondary electron (SE) images, left column) and signal distribution (backscattered electron images (BSE), right column). A SEM at $30 \mathrm{kV}$ of labeled barley chromosomes shows a signal gradient climaxing in the pericentric region and a signal gap corresponding to the primary constriction. Outer lateral fibrils at the primary constriction are unlabeled (arrows). B Acrocentric chromosomes from Vicia faba also exhibit a signal concentration at the pericentric regions symmetrical to the primary constriction. C, D On small acrocentric chromosomes of Oziroë biflora the entire short arm is labeled (C, right); independent of chromosome length, all signal regions were approximately equal in size (D, circles). Small chromosomes are not entirely labeled and suggest an axial symmetry which may define the functional centromere (D, right).
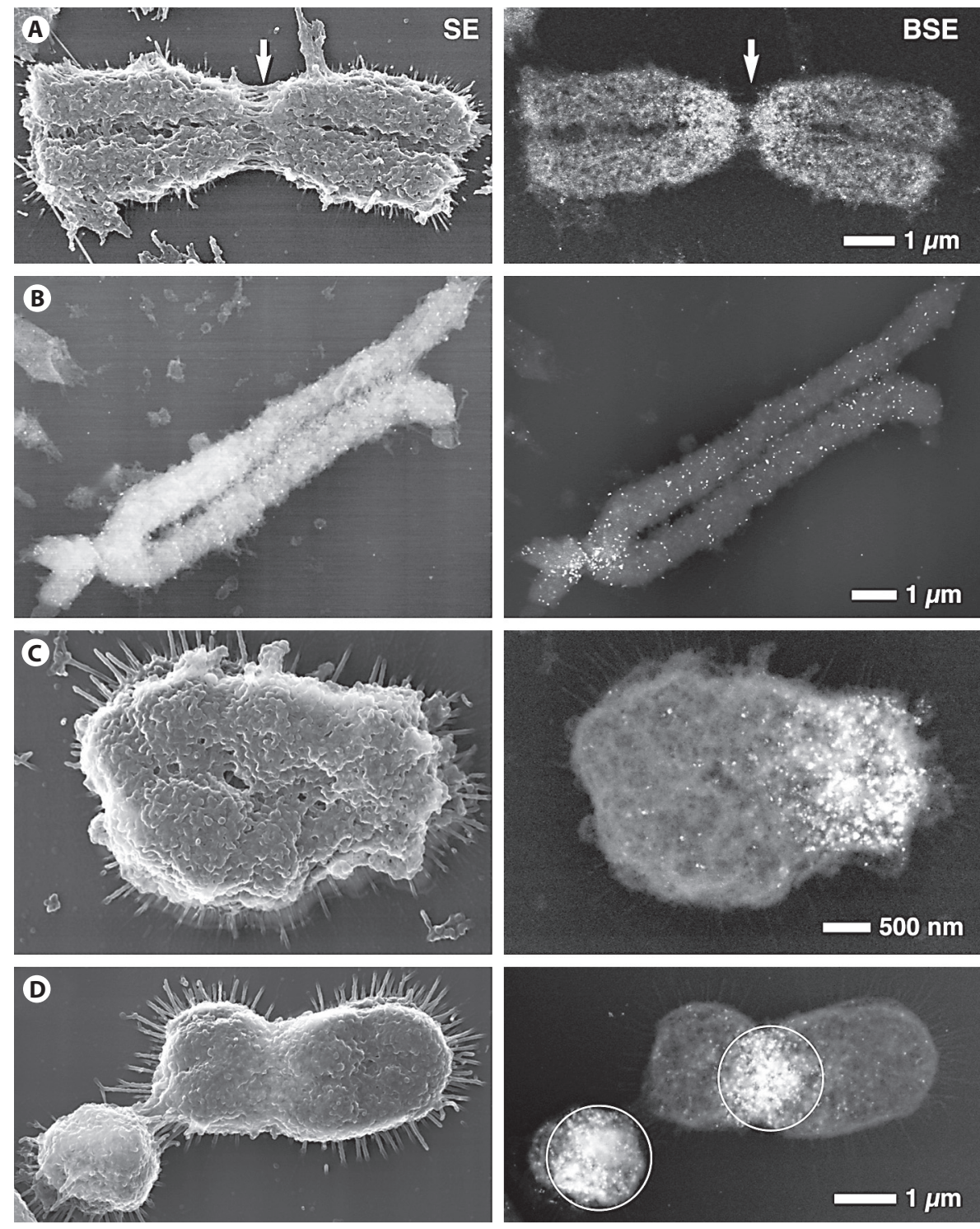

exhibited only trace labels for H3S10ph on the terminal tapered end of the chromosome.

The centromere-specific histone variant $\mathrm{CENH} 3$ was investigated in barley and in the wheat-barley addition line. For both species, labeling was strong at the centromere, with only negligible labels at the chromosome arms (fig. 5). In metacentric chromosomes, 2 strongly labeled regions, obviously for each chromatid, are located laterally on the primary constriction, clearly separated by a longitudinal signal gap (fig. 5A, B). Only few labels are located on the chromosome surface, whereas the majority of labels are located in the interior of the centromere and form a diffuse signal 'cloud' (fig. 5B). In the telocen- tric chromosomes of the wheat-barley addition line, there were only very few labels located terminally on the tapered end of the chromosome (fig. 5C).

\section{Chromosome Tomography with FESEM/FIB}

The interior centromere structure for barley chromosomes could be investigated with a novel application of a two-beam low voltage FESEM/FIB system. SE and BSE images were recorded at $2-3 \mathrm{kV}$. Sequential removal of material slices from whole mount chromosomes directly exposed chromosome interior; serial data were acquired by detection of secondary electrons (SE) and/or backscattered electrons (BSE) and were used as data sets for 
Fig. 5. Scanning electron micrographs of metacentric (cv. Steffi) and telocentric (wheat-barley addition line) barley chromosomes after immunogold labeling for CENH3, showing chromosome topography (secondary electron images (SE), left column) and signal distribution (backscattered electron images (BSE), right column). A Low magnification of a labeled barley chromosome reveals a clear specificity for the centromeric region. Two lateral signals are located at the centromere (right). B Higher magnification of the centromere (detail of $\mathbf{A}$, frame) shows that 2 diffuse lateral signals co-locate not only to the primary constriction (arrowheads), but also to directly bordering pericentric regions. Outermost lateral fibrils at the centromere are unlabeled. C On telocentric chromosomes from the wheat-barley addition line, only few labels for CENH3 are located terminally on the tapered end (circles).
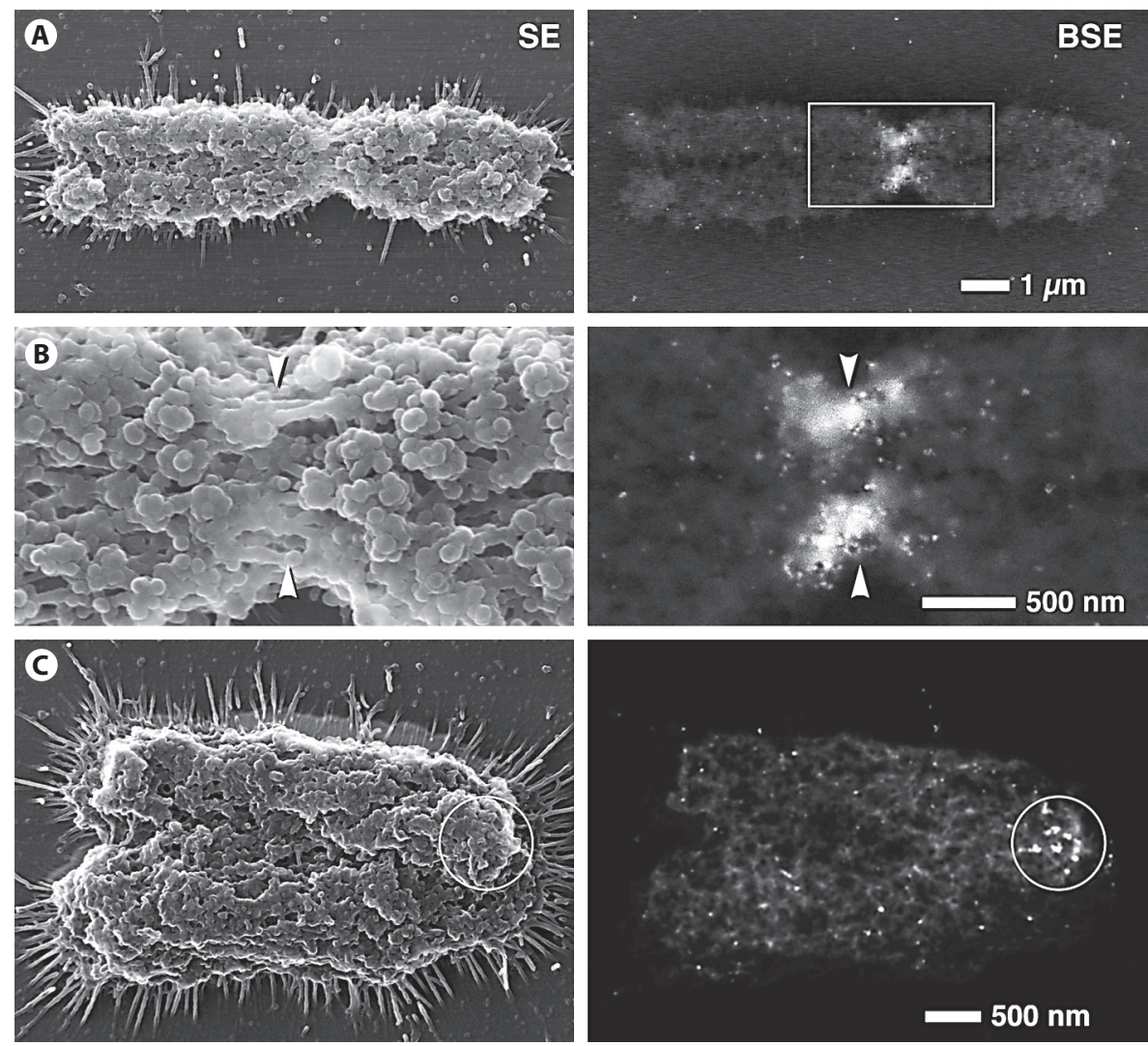

chromosome tomography. Since the section thickness determines the resolution of ultrastructural details, milling rates in the range of $10-30 \mathrm{~nm}$ were chosen to enable visualization of chromatin structures from $10 \mathrm{~nm}$ elementary fibrils to $30 \mathrm{~nm}$ solenoids and for detection of individual labels with an average diameter of $15 \pm 10$ $\mathrm{nm}$. With FIB milling, the chromosome interior visible at section surfaces could - for the first time - be structurally characterized as a network of compact chromatin regions, fibrillar structures and cavities (fig. 6, 7). In the interior of the centromere, longitudinal sections reveal parallel structures in the lateral centromere regions and compact chromatin regions, with little topographic contrast due to the smooth milled surface, interspersed with small cavities (figs. 6, 7). FIB data in barley indicate that the centromere contains a much less elaborate cavity network than the chromosome arms.

FESEM/FIB investigations also provided for the first time insight into the 3D spatial distribution of the H3S10ph and CENH3 in the chromosome interior. Serial longitudinal milling sections of barley chromosomes labeled with H3S10ph revealed that labels were distributed in a gradient along the entire metaphase chromosome, with maximum signal intensity in the extended pericentric region (fig. 6). In contrast to classical 'top-view' FESEM investigations at $30 \mathrm{kV}, \mathrm{H} 3 \mathrm{~S} 10$ ph label distribution in the majority of FESEM/FIB sections spanned the primary constriction in a narrow signal region. Outermost parallel fibrils are unlabeled (fig. 6).

Serial longitudinal FESEM/FIB milling sections of a mid-metaphase barley chromosome labeled for CENH3 exhibited a clear specificity for the centromeric region; markers in the chromosome arms are negligible (fig. 7). It could be shown with FESEM/FIB for the first time that $\mathrm{CENH} 3$ is distributed mainly in the interior of the centromere. In both longitudinal sections and cross-sections surprisingly few markers were located directly on the surface of the chromosome, but they rather were concentrated below a structurally compact cortex layer (fig. 7). Both longitudinal sections and cross-sections show that heavily labeled compact areas in the centromere are interrupted by few small, in some cases elongated, cavities (fig. 7). For CENH3, as for H3S10ph, the outermost parallel fibrils are unlabeled (fig. 7). 


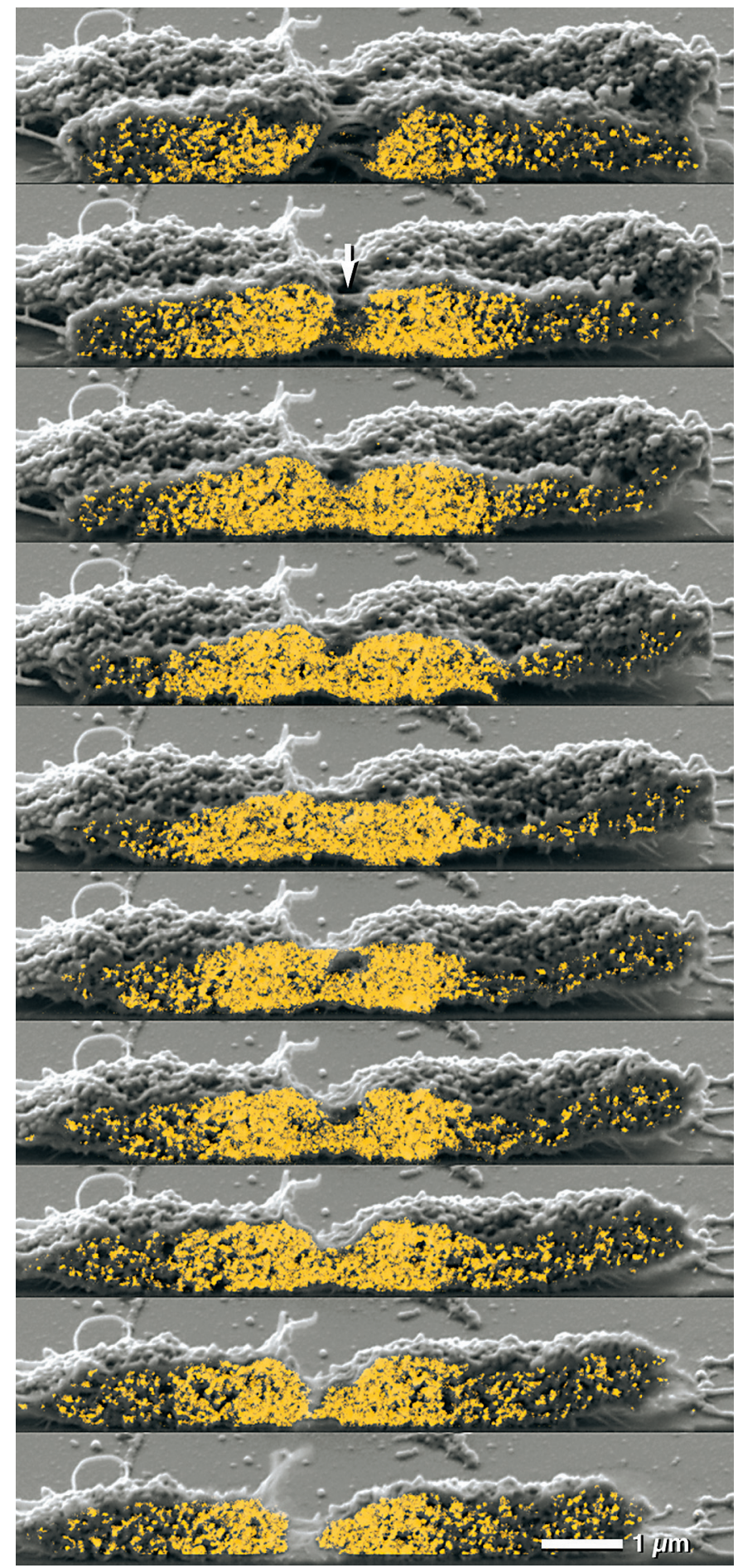

Fig. 6. Selected image series out of a total of 128 images of FESEM/ FIB sections ( $3 \mathrm{kV}$, section thickness $15 \mathrm{~nm}$ ) of barley chromosomes labeled for phosphorylated histone $\mathrm{H} 3$ at serine 10 (H3S10ph). Backscattered electron images (yellow) are superimposed on secondary electron images (greyscale). In contrast to

\section{Discussion}

A functional centromere is essential for stable and high-fidelity transfer of chromosomes to progeny cells in mitosis and meiosis. By definition the centromere is a structurally and genetically specific region in chromosomes responsible for kinetochore assembly and attachment of microtubules in the spindle apparatus [Black and Bassett, 2008]. Although centromeres have elusive species-specific DNA sequences, since they have a universal function, it follows that they also would have conserved structural elements.

When approaching the question whether there are ultrastructural characteristics that centromere variants have in common, it seems there is an exception for almost every generalization that can be made. The obvious example is the presence of a primary constriction. It is highly recognizable that a primary constriction, either interstitial or terminal, which is assumed to be a 'docking zone' for the kinetochore/microtubule complex, is characteristic for metaphase centromeres. According to our observations, there are some questions as to whether the presence of an interstitial constriction requires a minimum chromosome size. A lack of visible primary constriction would represent an economy of space and function for small chromosomes. This applies in particular to model organisms, for example Arabidopsis, which however still proves challenging for ultrastructural investigations. The lack of a primary constriction in holocentric chromosomes, here represented by Luzula spp., is well characterized [Braselton, 1971; Gernand et al., 2003; Haizel et al., 2005; Guerra et al., 2006]. In Luzula nivea putative centromeric DNA sequences have been located for light microscopy at local areas along the whole chromosome [Haizel et al., 2005]. One could speculate that the fibrillar network-like structures so often observed surrounding Luzula spp. chromosomes are residues of kinetochore elements and/or the spindle apparatus. Since SEM data show that there is no obvious sub-structural docking zone, it must be assumed that kinetochore and spindle apparatus assembly can be mediated by small

classical SEM data, direct viewing of chromosome interiors reveals that $\mathrm{H} 3 \mathrm{~S} 10$ ph labels (yellow) are distributed across the centromere in a narrow labeled region. Outermost lateral fibrils at the centromere are unlabeled (arrow). 

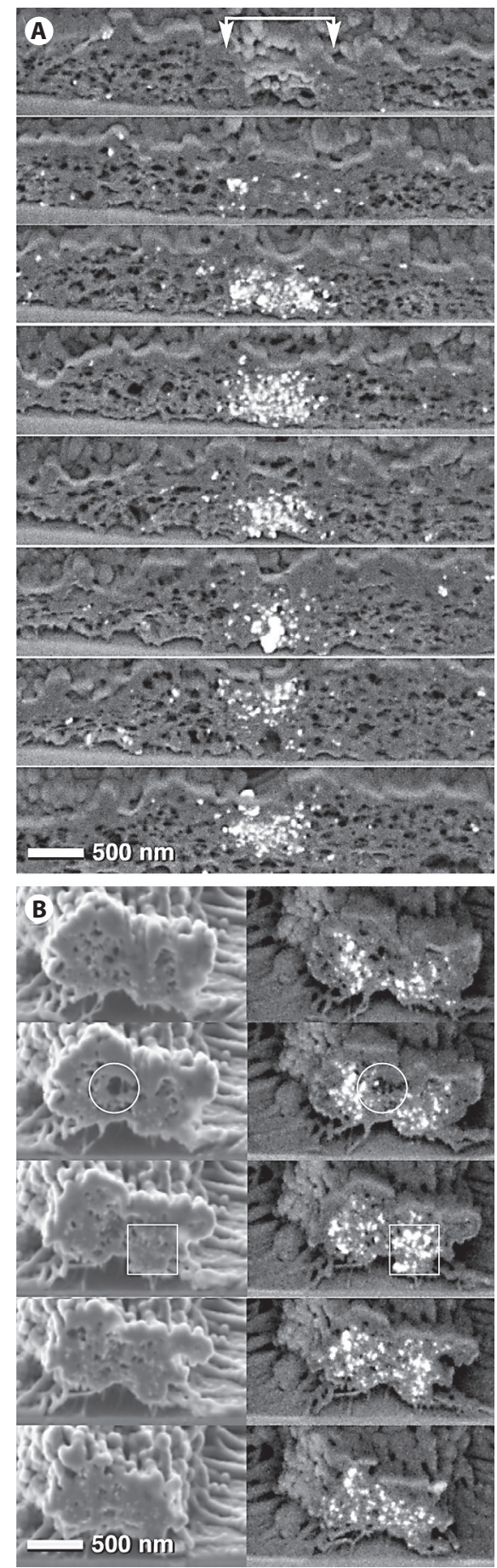

Fig. 7. FESEM/FIB section image series $(3 \mathrm{kV}$, section thickness $15 \mathrm{~nm}$; ordered from top to bottom) revealing direct insight into the chromosome interior and of spatial signal distribution of barley chromosomes labeled for the centromere-specific histone variant $\mathrm{CENH} 3$. A In longitudinal sections through a whole mount chromosome (centromeric region $=$ arrow) signals locate to subsurface loci specifically in the centromere (recorded in $20 \%$ SE:80\% BSE mode; selected images from every 4 th section out of local attachment areas, perhaps local 'constrictions' in a broad sense. Polarity of attachment sites along the chromosome arms, as suggested by light microscopic investigations [Nagaki et al., 2005], is supported by an obvious parallelity of sister chromatids in Luzula in early metaphase (fig. 3B). Indeed, light microscopic studies suggest that holocentric centromeres of $L$. nivea form a lateral groove [Nagaki et al., 2005]; although ongoing investigations are in progress, conclusive structural evidence for a lateral groove has yet to be established.

General ultrastructural characteristics of centromeres described previously include smaller chromomeres than on chromosome arms and parallel matrix fibrils, which play a key role in linear chromosome condensation [Wanner and Formanek, 2000]. The FESEM/FIB data presented here prove the presence of 'free space' that represents a cavity network which seems to be less elaborate in the centromere than in chromosome arms. The significance of this network, and particularly the compaction in the centromere, will require further FIB investigations including other types of chromosomes, protein, and DNA staining and cryo-fixation/cryo-SEM.

Until now, due to a predominance of exposed parallel fibers at the centromere in the organisms routinely studied (human, rye, and barley in particular), it was considered as a likely possibility that they are a characteristic feature of all centromeres [Harrison et al., 1982; Allen et al., 1988; Sumner, 1991, 1998; Wanner et al., 1991; Zoller et al., 2004]. The frequent observation in different organisms of centromeres without exposed parallel fibrils indicates, however, that these are not essential for centromere function. We assume, according to the Dynamic Matrix Model for chromosome condensation [Wanner and Formanek, 2000], that parallel matrix fibers are integral to chromosome architecture, but only exposed under certain conditions. These conditions include inhibiting formation of the spindle apparatus by arresting of chromosomes [Wanner and Schroeder-Reiter, 2008], isolation techniques (centrifugation, spreading of chromosomes) [Wanner et al., 2005], and, according to the data

a selected sector in a series of 78 images). Chromosome arms exhibit compact chromatin regions, fibrillar structures, and cavities. B Selected images from a cross-section series of 200 images through the centromeric region with separate SE and BSE detection shows that strongly labeled regions correspond with rather compact chromatin (frames), whereas signal-free areas correspond to cavities (circles). Labels are located in the interior of the centromere (right). 
presented here, size of chromosomes. The size of chromosomes would logically affect any physical forces during condensation, isolation, and spreading, in particular as observed in suspension preparations for which the influence of centrifugal forces are apparent (fig. 2C, D) [Wanner et al., 2005]. There is also evidence in wallaby (marsupial) hybrids that the length of the primary constriction is related to the number of centromere-specific DNA sequence repeats [Metcalfe et al., 2007]. It has been shown that some chromosomes have highly amplified centromere sequences and exhibit extremely long centromeric constrictions, in which both parallel fibrils and chromomeres are present [Metcalfe et al., 2007]. Characterization of centromere repeats in different species should help to determine whether structural differences are influenced by the amount of centromeric DNA.

Understanding the structure of centromeres is fundamental to the understanding of kinetochore assembly, spindle apparatus formation, and the modulation of chromosome dynamics. For 3D structural analysis with FESEM, the priority for chromosome isolation methods leans heavily on high yield and highest possible structural preservation of the chromosomes themselves, and investigation of their interactions with extra-chromosomal elements (e.g., kinetochore proteins or microtubules) with high resolution is difficult in routine. Even under conditions conducive to maintenance of spindle elements, putative spindle structures are rarely observed (fig. 1J, 3E). In these cases, however, the majority of fibrillar structures attach to bordering pericentric chromomeres, suggesting that specific spindle attachment sites are not as locally concentrated at the primary constriction as expected. This would be mechanically favorable when speculating that the traction forces during anaphase act upon the more stable chromosome arms instead of the implicitly weaker constriction, which could act as an elastic 'hinge', creating the $\mathrm{V}$-shape that reduces resistance in the viscous nucleoplasm. In this context, the concentrated pericentric localization of H3S10ph in plants at metaphase may be significant. It has been discussed that H3S10ph and its interaction with other chromosomal proteins could provide mechanical stability at the centromere prior to anaphase [Houben et al., 1999]. Data presented here show that within a chromosome complement there is also a uniformity of size of the labeled region which is independent of the overall size of chromosomes, suggesting that mechanical conditions prior to and during anaphase are similar for each chromosome, independent of its size.

Since H3S10ph is involved in sister chromatid cohesion [Kaszás and Cande, 2000; Manzanero et al., 2000;

Centromere Structure Analyzed with SEM and FIB
Gernand et al., 2003], it is arguable that the labeling gradient in barley with a concentration in the pericentric region reflects the degree of sister chromatid cohesion. Deducing from classical top-view SEM with $30 \mathrm{kV}$, the striking signal gap detected at the primary constriction (fig. 4A) would imply only weak cohesion. The interpretation of the extent of the signal gap depends on the physical properties of the labeled chromosomes which primarily influence back-scattered electron (BSE) detection. In principal, BSEs with energies of $30 \mathrm{keV}$ should easily be detected when emerging from depths of $<3 \mu \mathrm{m}$ within an organic matrix. However, the inclusion of heavy metal markers in different sizes, concentrations, and depths complicates the interpretation of the origin of signals which exhibit a wide range of sizes, contrast, brightness, and geometric definition. Surprisingly, direct insight into the chromosome interior with FESEM/FIB showed that there are indeed more H3S10ph labels than previously deduced from top-view SEM (compare figs. 4 and 6). This novel data achieved by direct detection of exposed interior signals indicate that $\mathrm{H} 3 \mathrm{~S} 10 \mathrm{ph}$ could also contribute to sister chromatid cohesion directly in the centromere. This further supports the speculation that $\mathrm{H} 3 \mathrm{S10ph}$ is involved in additional functions such as kinetochore orientation or spindle assembly.

Molecular and cytological studies have shown that CENH3 is an integral foundation for kinetochore proteins [Talbert et al., 2002, 2004; Black and Bassett, 2008], and exhibits 2 loci directly at the primary constriction that are assumed, according to current models, to be located at the outermost lateral border of the centromere [Blower et al., 2002; Black and Bassett, 2008]. Recent SEM data showed that outermost structures of the centromere are unlabeled for canonical histone $\mathrm{H} 3, \mathrm{H} 3 \mathrm{~S} 10 \mathrm{ph}$, and CENH3 [Houben et al., 2007]. In this study, using FESEM/FIB it could be shown for the first time that $\mathrm{CENH} 3$ is clearly located in the interior of the centromere, supporting the postulation that it is a core centromeric element, but challenging the postulation that it represents the outer centromere and is freely accessible for kinetochore and spindle assembly.

FESEM/FIB has proven to be a promising technique for combining molecular, cytological, and high resolution ultrastructural investigations. Further experiments are planned to combine FIB with established analytical methods for SEM, including alternative fixation procedures such as high-pressure freezing combined with cryo-SEM and in situ investigations, to contribute to the understanding of the complicated spatial puzzle that the centromere still presents. 


\section{Acknowledgements}

The authors gratefully acknowledge Sabine Steiner, Claudia Bubenzer-Hange, and Cornelia Niemann for excellent technical assistance. Materials from Paul Talbert, Andreas Houben, Jürke Grau, Takashi Endo, Jan Murken, and Rachel O’Neill are greatly appreciated. Work on FESEM/FIB system was made possible by Carl Zeiss, Oberkochen, Germany, in particular Fabian PérezWillard and Ulrike Zeile. This work was supported in part by a grant from the Deutsche Forschungsgemeinschaft (DFG; SCHR $1157 / 2-1)$

\section{References}

Allen TD, Jack EM, Harrison CJ: The three dimensional structure of human metaphase chromosomes determined by scanning electron microscopy, in Adolph KW (ed): Chromosomes and Chromatin, Vol 2, pp 51-72 (CRC Press, Boca Raton 1988).

Black BE, Bassett EA: The histone variant CENP$A$ and centromere specification. Curr Opin Cell Biol 20:91-100 (2008).

Blower MD, Sullivan BA, Karpen GH: Conserved organization of centromeric chromatin in flies and humans. Dev Cell 2:319-330 (2002).

Braselton JP: The ultrastructure of the non-localized kinetochores of Luzula and Cyperus. Chromosoma 6:89-99 (1971).

Drobne D, Milani M, Leser V, Tatti F, Zrimec A, et al: Imaging of intracellular spherical lamellar structures and tissue gross morphology by a focused ion beam/scanning electron microscope (FIB/SEM). Utramicroscopy 108:663-670 (2007).

Earnshaw WC: Mitotic chromosome structure. Bioessays 9:147-150 (1988).

Gernand D, Demidov D, Houben A: The temporal and spatial pattern of histone $\mathrm{H} 3$ phosphorylation at serine 28 and serine 10 is similar in plants but differs between mono- and polycentric chromosomes. Cytogenet $\mathrm{Ge}$ nome Res 101:172-176 (2003).

Guaglianone ER, Arroyo-Leuenberger S: The South American genus Oziroe (Hyacinthaceae-Oziroeoideae). Darwiniana 40:61-76 (2002).

Guerra M, Brasileiro-Vidal AC, Arana P: Mitotic microtubule development and histone $\mathrm{H} 3$ phosphorylation in the holocentric chromosomes of Rhynchospora tenuis (Cyperaceae). Genetica 126:33-41 (2006).

Haizel T, Lim YK, Leitch AR, Moore G: Molecular analysis of holocentric centromeres of Luzula species. Cytogenet Genome Res 109: 134-143 (2005).

Harrison CJ, Allen TD, Britch M, Harris R: High-resolution scanning electron microscopy of human metaphase chromosomes. J Cell Sci 56:409-422 (1982).

Houben A, Wako T, Furushima-Shimogawara R, Presting G: The cell cycle dependent phosphorylation of histone $\mathrm{H} 3$ is correlated with the condensation of plant mitotic chromosomes. Plant J 18:675-679 (1999).
Houben A, Schroeder-Reiter E, Nagaki K, Nasuda $\mathrm{S}$, Wanner $\mathrm{G}$, et al: CENH3 interacts with the centromeric retrotransposon cereba and GC-rich satellites and locates to centromeric substructures in barley. Chromosoma 116:275-283 (2007).

Kaszás É, Cande WZ: Phosphorylation of histone $\mathrm{H} 3$ is correlated with changes in the maintenance of sister chromatid cohesion during meiosis in maize, rather than the condensation of chomatin. J Cell Sci 113: 3217-3226 (2000).

Knott G, Marchman H, Wall D, Lich B: Serial section scanning electron microscopy of adult brain tissue using focused ion beam milling. J Neurosci 28:2959-2964 (2008).

Manzanero S, Arana PP, Maria J, Houben A: The chromosomal distribution of phosphorylated histone $\mathrm{H} 3$ differs between plants and animals at meiosis. Chromosoma 109:308-317 (2000).

Martin R, Busch W, Herrmann RG, Wanner G: Efficient preparation of plant chromosomes for high-resolution scanning electron microscopy. Chromosome Res 2:411-415 (1994).

Martin R, Busch W, Herrmann RG, Wanner G: Changes in chromosomal ultrastructure during the cell cycle. Chromosome Res 4: 288-294 (1996).

Metcalfe CJ, Bulazel KV, Ferreri GC, SchroederReiter E, Wanner G, et al: Genomic instability within centromeres of interspecific marsupial hybrids. Genetics 177:2507-2517 (2007).

Nagaki K, Cheng Z, Ouyang S, Talbert PB, Kim $\mathrm{M}$, et al: Sequencing of rice centromere uncovers active genes. Nat Genet 36:138-145 (2004).

Nagaki K, Kashihara K, Murata M: Visualization of diffuse centromeres with centromere-specific histone $\mathrm{H} 3$ in the holocentric plant Luzula nivea. Plant Cell 17:1886-1893 (2005).

Nasuda S, Hudakova S, Schubert I, Houben A, Endo TR: Stable barley chromosomes without centromeric repeats. Proc Natl Acad Sci USA 102:9842-9847 (2005).
Schroeder-Reiter E, Houben A, Wanner G: Immunogold labeling of chromosomes for scanning electron microscopy: a closer look at phosphorylated histone $\mathrm{H} 3$ in mitotic metaphase chromosomes of Hordeum vulgare. Chromosome Res 11:585-596 (2003).

Schroeder-Reiter E, Houben A, Grau J, Wanner G: Characterization of a peg-like terminal NOR with light microscopy and high resolution scanning electron microscopy. Chromosoma 115:50-59 (2006).

Schubert I, Dolezel J, Houben A, Scherthan H, Wanner G: Refined examination of plant metaphase chromosome structure at different levels made feasible by new isolation methods. Chromosoma 102:96-101 (1993).

Sumner AT: Scanning electron microscopy of mammalian chromosomes from prophase to telophase. Chromosoma 100:410-418 (1991).

Sumner AT: The structure of the centromeric region of $\mathrm{CHO}$ chromosomes. Cell Biol Int 22: 127-130 (1998).

Talbert PB, Masuelli R, Tyagi AP, Comai L, Henikoff S: Centromeric localization and adaptive evolution of an Arabidopsis histone $\mathrm{H} 3$ variant. Plant Cell 14:1053-1066 (2002).

Talbert PB, Bryson TD, Henikoff S: Adaptive evolution of centromere proteins in plants and animals. J Biol 3:18 (2004).

Wanner G, Formanek H: Imaging of DNA in human and plant chromosomes by high-resolution scanning electron microscopy. Chromosome Res 3:368-374 (1995).

Wanner G, Formanek H: A new chromosome model. J Struc Biol 132:147-161 (2000).

Wanner G, Schroeder-Reiter E: Scanning electron microscopy of chromosomes. Methods Cell Biol 88:451-474 (2008).

Wanner G, Formanek H, Martin R, Herrmann RG: High resolution scanning electron microscopy of plant chromosomes. Chromosoma 100:103-109 (1991).

Wanner G, Schroeder-Reiter E, Formanek H: 3D analysis of chromosome architecture: advantages and limitations with SEM. Cytogenet Genome Res 109:70-78 (2005).

Zoller JF, Herrmann RG, Wanner G: Chromosome condensation in mitosis and meiosis of rye (Secale cereale L.). Cytogenet Genome Res 105:134-144 (2004). 